\title{
Third-Generation TKI Resistance Due to SCLC Transformation: A Case Report and Brief Review
}

This article was published in the following Dove Press journal: OncoTargets and Therapy

\author{
Shen Chen (iD) \\ Yayi $\mathrm{He} \mathbb{D}^{\prime}$ \\ Juan $\mathrm{Liu}^{2}$ \\ Xiaoxia Chen' \\ Jia $\mathrm{Yu}^{\prime}$ \\ Wei $\mathrm{Li}^{\prime}$ \\ Bin Chen' \\ Chenglong Sun ${ }^{3}$ \\ Caicun Zhou' \\ 'Department of Medical Oncology, \\ Shanghai Pulmonary Hospital, Tongji \\ University Medical School Cancer \\ Institute, Tongji University School of \\ Medicine, Shanghai, People's Republic of \\ China; ${ }^{2}$ Department of Respiratory, \\ Shanghai Fourth People Hospital, \\ Shanghai, People's Republic of China; \\ ${ }^{3}$ Department of Medical Oncology, Anhui \\ No.2 Provincial People's Hospital, Anhui, \\ People's Republic of China
}

Objective: We reported a case of pathologic type transformed from adenocarcinoma to small-cell lung cancer (SCLC) after being treated with third-generation epidermal growth factor receptor tyrosine kinase inhibitor (EGFR-TKI).

Materials and methods: The lung cancer pathologic type was transformed from adenocarcinoma to SCLC after the treatment of third-generation EGFR-TKI for 4.3 months. Four times of lung biopsies were conducted at a different time and different lesions. And the patient was treated with a variety of regimens after SCLC transformation, including etoposide combined with carboplatin (EC), irinotecan combined with oxaliplatin (IO), Abraxane, and Apatinib. These treatments got a good response, but quick progression. We also monitored the dynamic change of serum neuron-specific enolase (NSE) and pro-gastrin releasing peptide (Pro-GRP).

Results: Adenocarcinoma could transform into SCLC after the treatment of TKI. Tumor cells were heterogeneity, so adenocarcinoma and SCLC could be co-existed. The fluctuation of the NSE was consistent with the response of treatment and the progression of the tumor. Conclusion: Regimens for the primary SCLC were also available for the transformed SCLC, but the duration of the response was short. NSE could be an effective biomarker for dynamic monitoring of the efficacy in SCLC transformation.

Keywords: epidermal growth factor receptor tyrosine kinase inhibitor, EGFR-TKI, smallcell lung cancer, SCLC, pathological transformation, resistance

\section{Introduction}

Lung cancer is the most common cancer type. Epidermal growth factor receptor $(E G F R)$ has been recognized as a remarkable driver gene in lung adenocarcinoma. Epidermal growth factor receptor tyrosine kinase inhibitors (EGFR-TKIs) have achieved significant effects in $E G F R$-positive mutant lung cancer. Normally, acquired resistance invariably happened in about 1 year after EGFR-TKIs. ${ }^{1}$ The secondary T790M mutation plays a key role in up to $50 \%$ first-generation EGFR-TKI resistance cases. ${ }^{2}$ Third-generation EGFRTKI is a powerful drug against these cases. ${ }^{3}$ Unfortunately, drug resistance is also inevitable. The mechanisms are not entirely clear. One mechanism is the transformation of adenocarcinoma to SCLC. ${ }^{4}$ The incidence of SCLC transformation is low.

Here, we reported a case of third-generation EGFR-TKI resistance due to SCLC transformation.

\section{Case Presentation}

A 49-year-old man with no smoking history detected a lesion in left lower lobe lung by X-ray during an annual checkup on 8th May 2013. A curative operation was
Correspondence: Caicun Zhou Department of Medical Oncology, Tongji University Medical School Cancer Institute, Tongji University School of Medicine, No. 507 Zhengmin Road, Shanghai 200433, People's Republic of China

Email caicunzhoudr@163.com 
performed in Shanghai pulmonary hospital on third Jun 2013. The laboratory findings were normal, including the carcinoembryonic antigen (CEA), neuron-specific enolase (NSE) and pro-gastrin releasing peptide (Pro-GRP). Pathological findings were as follows, the tumor was $4.5 \mathrm{~cm} \times 3 \mathrm{~cm} \times 3.2 \mathrm{~cm}$, moderately differentiated adenocarcinoma (Figure 1A), 6th and 11th hilar mediastinal lymph nodes metastasis and EGFR exon 21 L858R mutant.

Adjuvant chemotherapy regimen of docetaxel combined with cisplatin was conducted for 4 cycles after the surgery. The patient refused adjuvant radiotherapy. The disease was relapsed and metastasized on 5th Jan 2014. CT scans showed multiple tiny nodules distributed in both lungs. He started to receive first-generation EGFR-TKI (gefitinib). The disease was progressed on 5th May 2015. A ct-guided needle biopsy was conducted to the lesion of the right lung. The result showed adenocarcinoma (Figure 1B) with EGFR L858R and T790M mutant. Followed with 2 cycles of chemotherapy of pemetrexed combination with cisplatin, disease was progression on 2nd Aug 2015. Then, third-generation EGFR-TKI (osimertinib) was administered on 17th Sep 2015. The best efficacy evaluation was a partial response (PR). He got a progression disease (PD) on 27th Jan 2016. We conducted a CT-guided needle aspiration in the enlarged lesion in his right lung (Figure 2A).
Pathology (29th Jan 2016) showed poorly differentiated carcinoma, SCLC according to immunohistochemistry (Napsin A -, TTF 1 +, Syn +) (Figures $1 \mathrm{C}$ and 3A). NSE was elevated $(38.48 \mathrm{ng} / \mathrm{mL}$, normal value range: 0 $20 \mathrm{ng} / \mathrm{mL}$ ) (Figure 4). Etoposide combined with carboplatin (EC) was administered. CT scan showed a remarkable shrinkage of the tumor. Response evaluation was PR (after 2 cycles) (Figure 5A). The response evaluation of 4 cycles was PD (Figure 5B). NSE was elevated to $45.35 \mathrm{ng} / \mathrm{mL}$ (Figure 4). We replaced the EC regimen to irinotecan combined with oxaliplatin (IO), and got PR after 2 cycles (Figure 5C). NSE was decreased to the normal range $(18.17 \mathrm{ng} / \mathrm{mL})$ (Figure 4). The disease was progressed after 4 cycles on 19th Oct 2018 (Figure 5D). NSE was increased to $52.90 \mathrm{ng} / \mathrm{mL}$ (Figure 4).

We conducted a CT-guided needle biopsy in two different sites (Figure 2B and C). Interestingly, the result showed that one was SCLC (IHC: Napsin A-, TTF-1+, Syn-, CD 56+) (Figures 1D and 3B), and the other was lung adenocarcinoma (IHC: Napsin A+, TTF-1+) (Figures 1E and 3C).

Abraxane was administered to the subjects after the IO regimen failed and obtained an inspiring efficacy, most lesions exhibited shrinkage (Figure 5E). NSE was returned to the normal value $(18.17 \mathrm{ng} / \mathrm{mL})$ (Figure 4$)$. Unfortunately, the duration of the response was short. Lesions were enlarged after 4 cycles of Abraxane. Chest
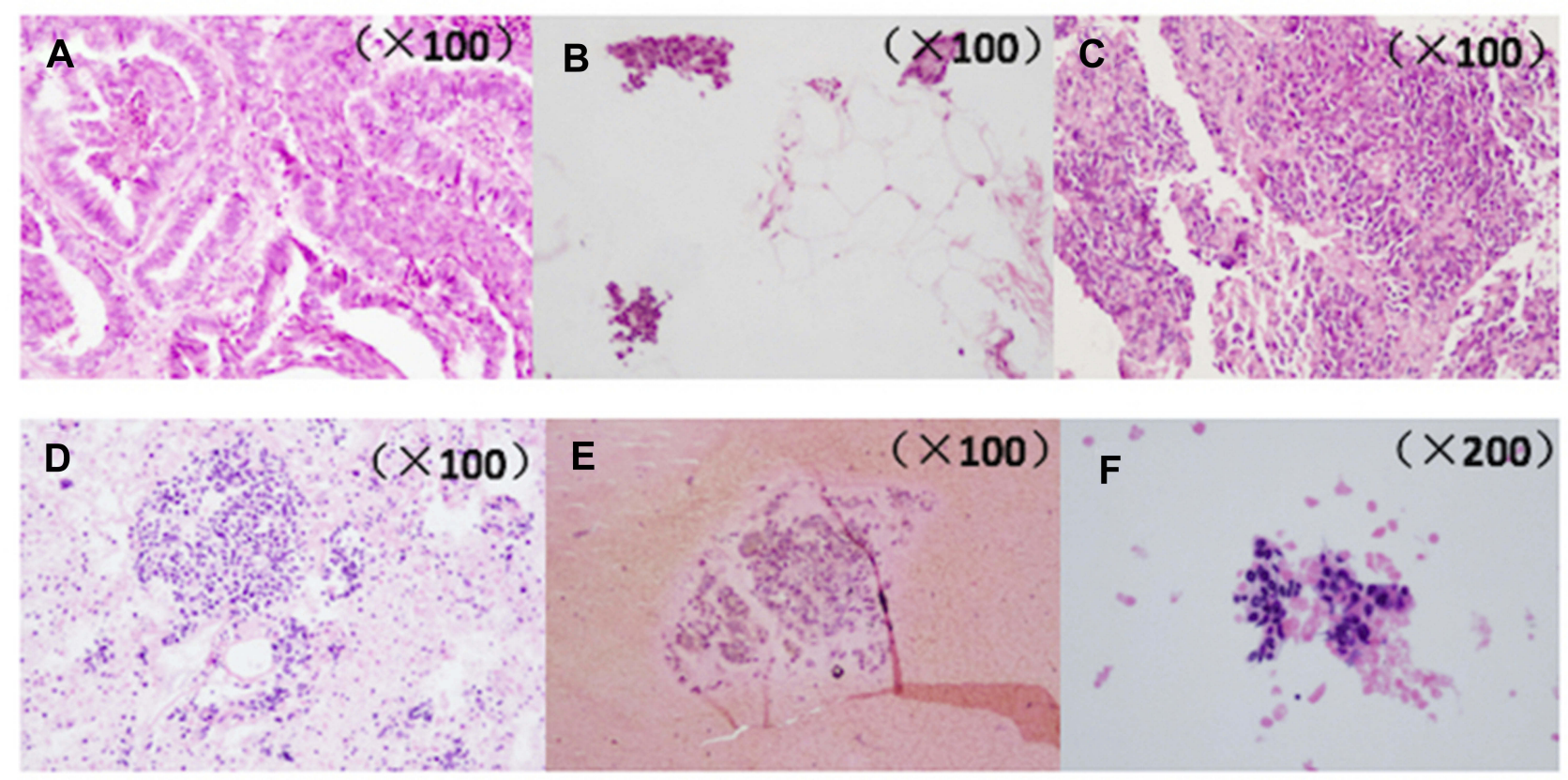

Figure I The change of pathological morphology. (A) Jun 2013, postoperative specimens: moderately differentiated adenocarcinoma. (B) Aug 20I5, resistant to gefitinib: moderately differentiated adenocarcinoma. (C) Jan 2016, resistant to AZD929I: poorly differentiated carcinoma. (D) Oct 2016, lesion A resistant to EC/IO, SCLC. (E) Oct 2016, lesion B resistant to EC/IO, LADC. (F) April 2017, enlarged lesion of right lung after apatinib, NSCLC. 

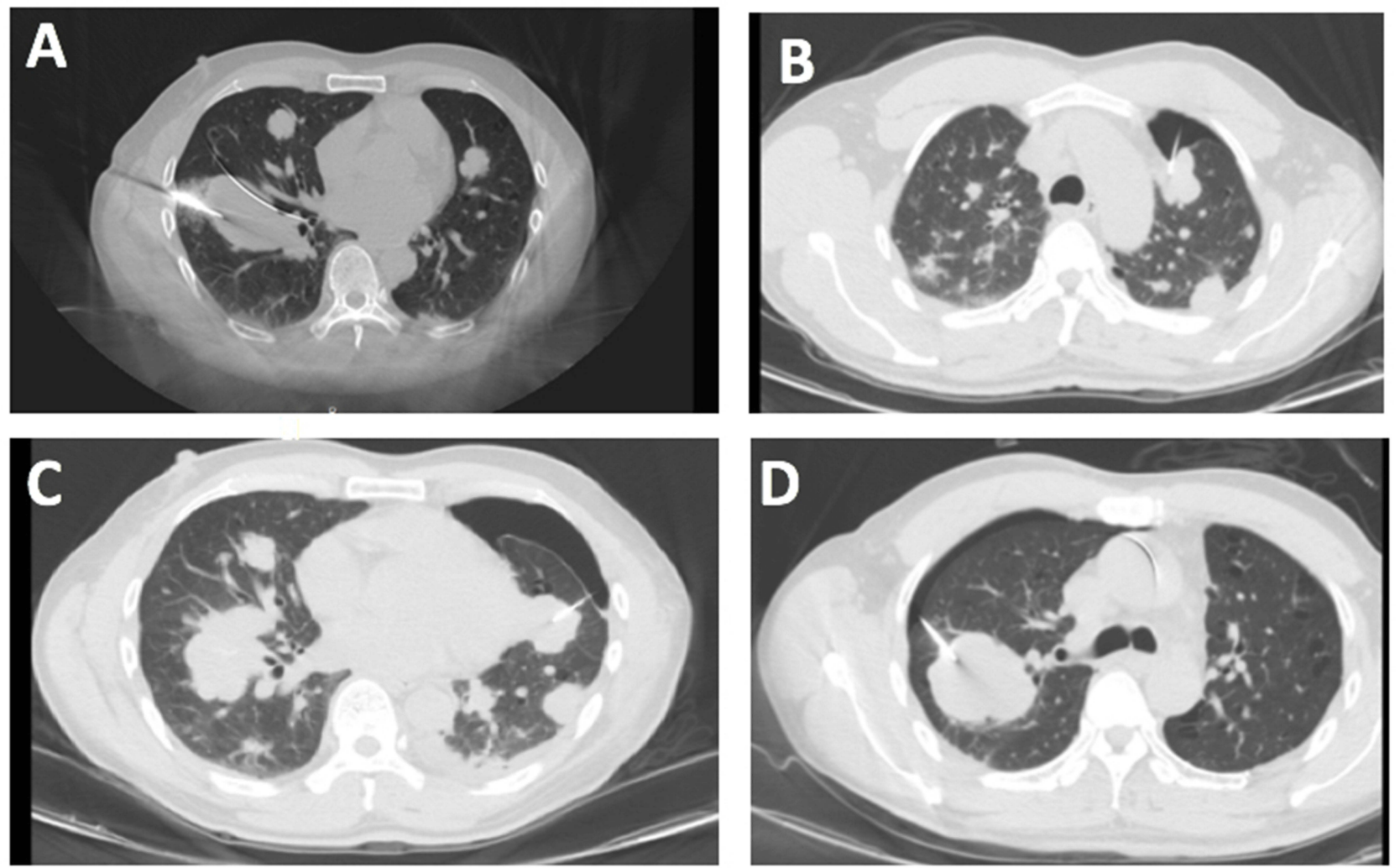

Figure 2 Four times CT-guide needle biopsy. (A) Jan 2016, CT-guided needle biopsy in the enlarged lesion in his right lung. (B) Oct 2016, CT-guided needle biopsy in lesion A after resistant to IO. (C) Oct 2016, CT-guided needle biopsy in lesion B after resistant to IO. (D) Apr 20I7, CT-guided needle biopsy in the enlarged lesion of right lung after apatinib.

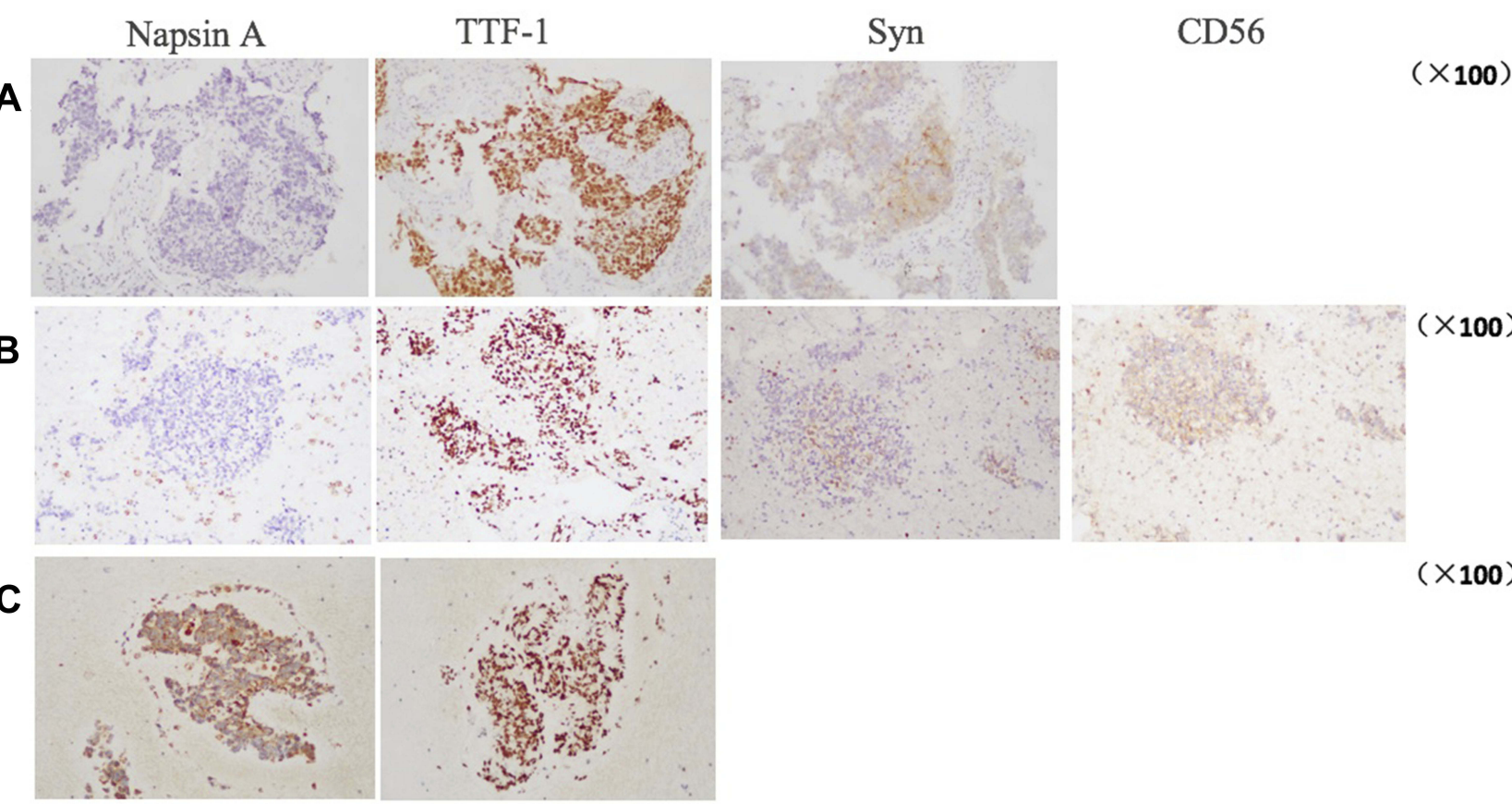

Figure 3 The change of Immunohistochemistry. (A) Jan 2016, resistant to AZD929I, indicated SCLC (IHC: NapsinA-, TTFI+, Syn+). (B) Oct 20I6, lesion A after resistant to IO, indicated SCLC (IHC: NapsinA-, TTF-I+, Syn-, CD56+). (C) Oct 2016, lesion B after reistance to IO, indicated adenocarcinoma (IHC: NapsinA+,TTF-I+). 

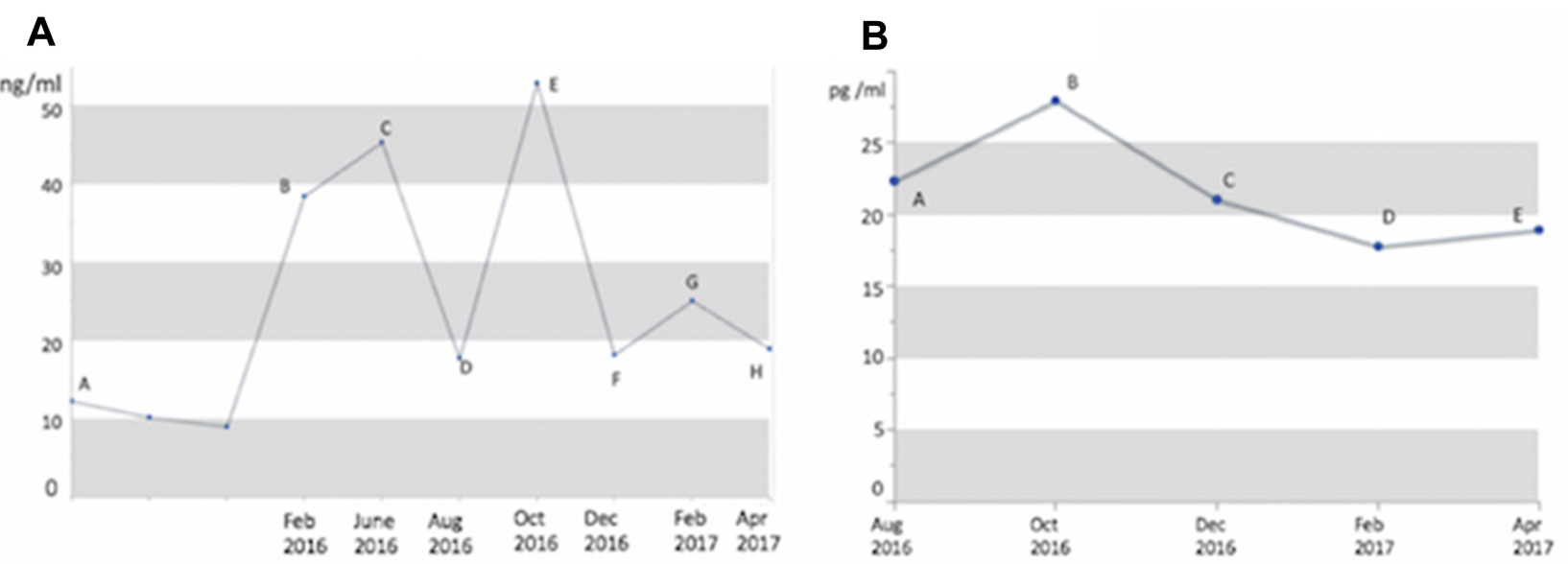

Figure 4 Fluctuation of serum NSE and pro-GRP. (A) The fluctuation of serum NSE (I2.26 ng/mL at baseline, $38.48 \mathrm{ng} / \mathrm{mL}$, at the time of resistant to AZD929I,45.35 $\mathrm{ng} / \mathrm{mL}$ at time of resistant to EC after 4 cycles, $17.7 \mathrm{I} \mathrm{ng} / \mathrm{mL}$ at time of response to $1 \mathrm{O}$ after 2 cycles, $52.9 \mathrm{ng} / \mathrm{mL}$ at time of resistant to $1 \mathrm{O}$ after $4 \mathrm{cycles}$, $18.17 \mathrm{ng} / \mathrm{mL}$ at time of response to abraxane after 4 cycles, $25.09 \mathrm{ng} / \mathrm{mL}$ at time of resistant to abraxane after 4 cycles, $18.93 \mathrm{ng} / \mathrm{mL}$ at time of treatment of apatinib after $6 \mathrm{weeks}$ ). (B) The fluctuation of serum pro-GRP $(22.35 \mathrm{pg} / \mathrm{mL}$ at time of response to $1 \mathrm{O}$ after 2 cycle, $27.94 \mathrm{pg} / \mathrm{mL}$ at time of resistant to 10 after $4 \mathrm{cycles}, 21.05 \mathrm{pg} / \mathrm{mL}$ at time of response to abraxane after 4 cycles, $17.79 \mathrm{pg} / \mathrm{mL}$ at time of resistant to abraxane after 4 cycles, $18.94 \mathrm{pg} / \mathrm{mL}$ at time of treatment of apatinib after 6 weeks).

CT showed disease progression again (Figure 5F). NSE was raised to $25.09 \mathrm{ng} / \mathrm{mL}$ (Figure 4). Apatinib, a type of small molecule vascular endothelial growth factor tyrosine kinase inhibitors (VEGF-TKIs), was administered to the patient. Amusingly, one month later, nearly all lesions were shrunk (Figure 5G), while one solitary lesion at the right lung was enlarged (Figure 5H). We conducted a CT-guided needle puncture in the enlarged lesion of the right lung (Figure 2D). The pathological type turned out to be NSCLC (Figure 1F). NSE was $18.93 \mathrm{ng} / \mathrm{mL}$ (Figure 4).

As the performance status (PS) score was 2, the patient received the best support care (BSC). He died of respiratory failure on 11th July 2017 (Figure 6).

\section{Discussion}

EGFR-TKIs achieved remarkable efficacy in clinical practice and was recognized as standard first-line therapy in NSCLC patients with EGFR sensitive mutation worldwide., ${ }^{5,6}$ Acquired resistance to TKI was an annoying problem. $^{7}$ T790M mutation occurred in nearly half of cases when patients were resistant to the first-generation EGFR-TKIs. Osimertinib was the standard therapy for NSCLC patients with T790M mutation. ${ }^{3}$ There were 3 resistant types of osimertinib: both the initial EGFR sensitive and T790M mutation were disappeared; $E G F R$ sensitive mutation could be detected, but T790M mutation was disappeared; both the initial sensitive mutation and T790M mutation existed. The mechanisms of resistance to osimertinib were various, including acquired C797S mutation, SCLC transformation, c-MET amplification, HER-2 amplification, BRAF mutation, KRAS mutation, and PI3KCA mutation, etc. The patient, we presented, harbored the first-generation EGFR-TKI as L858R and T790M mutation, who was resistant to the thirdgeneration $E G F R$-TKI because of SCLC transformation. It was reported that lung cancer patients harboring EGFR mutations were more likely to get SCLC transformation than EGFR-wild type patients. ${ }^{8}$ The median time of SCLC transformation was 17.8 months (14.3 to 26.2 months), ${ }^{4}$ while the time was 30.7 months in our case (from third Jun 2013 to 29th Jan 2016). In this case, the progressionfree survival (PFS) of the first-generation EGFR-TKI was 17.5 months (from 16th Jan 2014 to 30th Jun 2015), while the third-generation EGFR-TKI was administered for about 4.3 months (from 16th Sep 2015 to 24 Jan 2016). The resistance mechanism was probably an SCLC transformation during osimertinib therapy in this patient.

It was reported that SCLC transformation had a high incidence of never smoking, young, EGFR-positive mutant NSCLC patients. ${ }^{9}$ EC was the most commonly used chemotherapy regimen after transformation SCLC, it was reported that the estimated PFS was 3.4 months (2.4-5.4 months). ${ }^{4}$ The PFS was 3.8 months in our case (from third Feb 2016 to 26th May 2016), which was consistent with the published data. Furthermore, we presented the patient who has also accepted some available regimens, such as Abraxane and Apatinib. The efficacy as well, but the duration of the response was short. 

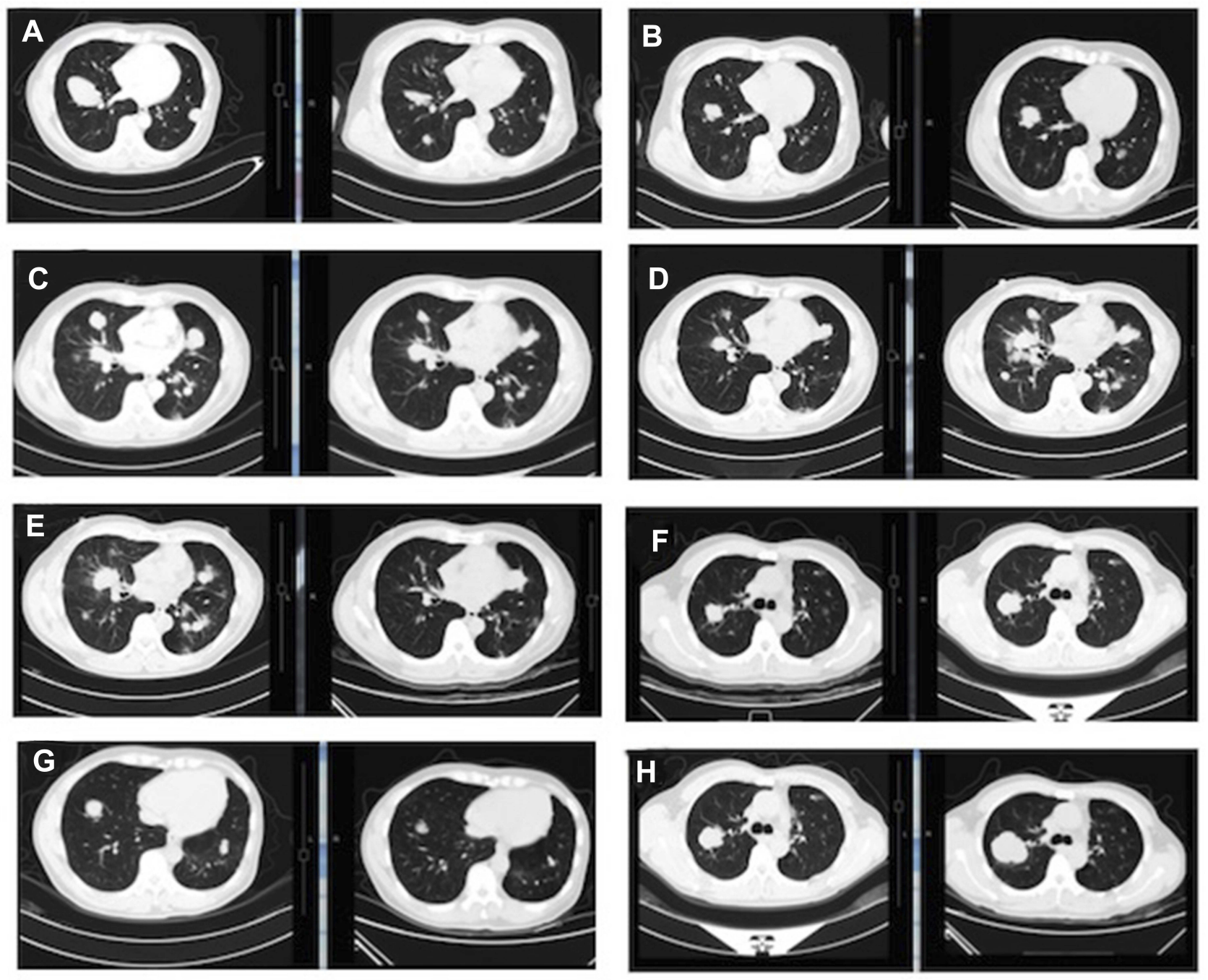

Figure 5 The change of chest CT after different regimens. (A) PR after 2 cycles of EC (right lower lobe, from 6.71 to $3.1 \mathrm{~cm}$ ). (B) PD after 4 cycles of EC (right lower lobe, from 2.9 to $3.9 \mathrm{~cm}$ ). (C) PR after 2 cycles of IO (left upper lobe, from 2.72 to I.65 cm). (D) PD after 4 cycles of IO (left upper lobe, from I.9 to $3.1 \mathrm{~cm}$ ). (E) PR after $2 \mathrm{cycles}$ of Abraxane (right lower lobe, from 5.41 to $0.4 \mathrm{~cm}$ ). (F) PD after 4 cycles of Abraxane (right upper lobe, from 2.8 to $4.0 \mathrm{~cm}$ ). (G) Shrunken lesion of left lung after Apatinib (left lower lobe, from 3.47 to $2.34 \mathrm{~cm}$ ). (H) Enlarged lesion at right lung after Apatinib (right upper lobe, from 3.74 to $5.73 \mathrm{~cm}$ ).

Serum NSE and pro-GRP were commonly recommended as a tumor biomarker for SCLC. Huang reported that proGRP and NSE were accurate biomarkers in evaluating response to the regimen and predicting the prognosis in patients with SCLC. ${ }^{10}$ Cavalieri reported that pro-GRP was available biomarker in the SCLC and sensitive for SCLC diagnosis at cutoff value of $77.8 \mathrm{pg} / \mathrm{L} .{ }^{11}$ Zhang reported that NSE increased 5-folds when adenocarcinoma transformed to SCLC. The result suggests that increased NSE levels may point to pathological transformation. ${ }^{12}$ Akira suggests that

Operation\&TP
Jun Gefitinib

Figure 6 Multiple treatment regimens.

Abbreviations: TP, docetaxel combined with cisplatin. AP, pemetrexed combination with cisplatin. EC, Etoposide combined with carboplatin. IO, irinotecan combined with oxaliplatin. BSC, best support care. ADC, adenocarcinoma. SCLC, small- cell lung cancer. 
pro-GRP shows a higher correlation to tumor reopens than NSE in primary SCLC. ${ }^{13}$ However, there were seldom references discuss the pro-GRP in transformed SCLC.

In our case, we observed the different fluctuations between them (Figure 4). Both NSE and pro-GRP were normal at the time of diagnosis. NSE showed a rapid increase on 6th Feb 2016, when the pathology was transformed to SCLC. The fluctuation of the NSE was consistent with the response of the treatment and the progression of the tumor. NSE could be an available and effective biomarker for transformation SCLC (Figure 4A). Pro-GRP was in a normal range during the whole course of the disease (Figure 4B). So we speculated that SCLC transformation had the potential difference from primary SCLC. More research should be done in the transformation SCLC.

Multiple treatment regimens were applied to this patient (Figure 6). EC therapy was the standard regimen of SCLC. ${ }^{14}$ In this case, the tumor had a moderate response after 2 cycles of treatment (Figure 5A), but it progressed after 4 cycles of treatment (Figure 5B). The duration of response was significantly shorter than that in primary SCLC. Similarly, the situation happened with irinotecan treatment (Figure 5C and D). Efficacy in SCLC transformation patients was poorer than that in primary SCLC patients. ${ }^{15}$ It was reported that bevacizumab combined with chemotherapy was effective in SCLC in firstline treatment. ${ }^{16,17}$ In our case, we found apatinib was available in patients with SCLC transformation (Figure 5E).

We also observed that the response among different lesions was different. We conducted needle biopsy in different lesions (Figure 2B and $\mathrm{C}$ ), the pathology results of 2 lesions were different: one was SCLC (Figures 1D and 3B), and the other was adenocarcinoma (Figures $1 \mathrm{E}$ and $3 \mathrm{C}$ ). SCLC and adenocarcinoma co-existed simultaneously. This was an interesting phenomenon. Lung adenocarcinoma and SCLC might exist at initial tumor lesions. Then, the proportion of lung adenocarcinoma cells decreased due to the EGFR-TKI treatment and the proportion of SCLC cells was increased. ${ }^{8}$ Re-biopsy and multiple lesions biopsy were important, especially when the efficacy was not good or different lesions presented discordant response. In this case, we conducted a lung biopsy of the right lung at enlarged lesion during the administered of Apatinib on 20th Apr 2017 (Figure 2D), and the lesion turned out to be NSCLC (Figure 1F). It might be related to the heterogeneity of the cancer cells. ${ }^{18}$

Previously report showed that activated retinoblastoma 1 (RB1) and tumor protein p53 (TP53) were correlated with EGFR-TKI resistant SCLC. RB1 losing was observed in
$100 \%$ of SCLC transformed cases. ${ }^{19}$ NSCLC patients presenting with TP53 and RB1 mutations at baseline are more likely to transform to SCLC. ${ }^{20}$ A hypothesis was that the transformed SCLC was evolved from adenocarcinoma or developed from the common precursor as adenocarcinoma. It was believed that EGFR mutation could promote phenotypic changing. ${ }^{9}$ TKI might activate the pluripotent cells or induce specific differentiation. The pathological change occurred more frequently under the selective pressure of TKI. Studies have revealed that the alveolar type II cells might be a common precursor of both lung adenocarcinoma and SCLC cells. ${ }^{8}$ EGFR-mutant lung cancer cells which evolved from alveolar type II cells might have the potential to transform into SCLC.

Additionally, the pathological transformation was existed not only in EGFR mutant lung adenocarcinoma patients but also detected in a patient treated with anaplastic lymphoma kinase (ALK)-TKI. The phenomenon was reported in patients treated with Crizotinib or Alectinib. It was also suggested that SCLC transformation was not unique in patients treated with TKI. Two cases of lung adenocarcinoma with $E G F R$-wild-type were reported. ${ }^{21}$ What's more? An $E G F R$-wild-type NSCLC patient transformed from NSCLC to SCLC during nivolumab (anti-programmed cell death protein 1 monoclonal antibody) was reported recently. ${ }^{22}$ The mechanisms of SCLC transformation were not clear. More research was needed to reveal the mechanisms. And the transformation SCLC shared the different characteristics with the primary SCLC, in the aspect of the tumor biomarkers, the effect of traditional treatment regimen and response. The changes in the level of blood tumor biomarkers, particularly for NSE in serum, might suggest the pathological transformation occurred; re-biopsy or multiple lesion biopsies were needed.

\section{Ethic and Consent Statements}

Written informed consent has been provided by the patient's son to have the case details and any accompanying images published. No institutional approval was required to publish the case details.

\section{Acknowledgments}

This study was supported in part by a grant from National Natural Science Foundation of China (81802255), Shanghai Pujiang Program (17PJD036) and a grant from Shanghai Municipal Commission of Health and Family Planning Program (20174Y0131), National Key Research \& Development Project (2016YFC0902300), Major Disease Clinical Skills 
Enhancement Program of Three Year Action Plan for promoting clinical skills and clinical innovation in municipal hospitals, Shanghai Shen Kang Hospital Development Center Clinical Research Plan of SHDC (16CR1001A), "Dream Tutor “Outstanding Young Talents Program (fkyq1901), Key Disciplines of Shanghai Pulmonary Hospital (2017ZZ02012), grant of Shanghai Science and Technology Commission (16JC 1405900). Shen Chen and Yayi He are co-first authors for this study.

\section{Disclosure}

The authors report no conflicts of interest in this work.

\section{References}

1. Engelman JA, Janne PA. Mechanisms of acquired resistance to epidermal growth factor receptor tyrosine kinase inhibitors in non-small cell lung cancer. Clin Cancer Res. 2008;14:2895-2899. doi:10.1158/ 1078-0432.CCR-07-2248

2. Yu HA, Arcila ME, Rekhtman N, et al. Analysis of tumor specimens at the time of acquired resistance to EGFR-TKI therapy in 155 patients with EGFR-mutant lung cancers. Clin Cancer Res. 2013;19:2240-2247. doi:10.1158/1078-0432.CCR-12-2246

3. Mok TS, Wu YL, Ahn MJ, et al. Osimertinib or platinum-pemetrexed in EGFR T790M-positive lung cancer. $N$ Engl $J$ Med. 2017;376: 629-640. doi:10.1056/NEJMoa1612674

4. Marcoux N, Gettinger SN, O'Kane G, et al. EGFR-mutant adenocarcinomas that transform to small-cell lung cancer and other neuroendocrine carcinomas: clinical outcomes. J Clin Oncol. 2019;37: 278-285. doi:10.1200/JCO.18.01585

5. Yang JC, Wu YL, Schuler M, et al. Afatinib versus cisplatin-based chemotherapy for EGFR mutation-positive lung adenocarcinoma (LUX-Lung 3 and LUX-Lung 6): analysis of overall survival data from two randomised, phase 3 trials. Lancet Oncol. 2015;16: 141-151. doi:10.1016/S1470-2045(14)71173-8

6. Zhou C, Wu YL, Chen G, et al. Erlotinib versus chemotherapy as first-line treatment for patients with advanced EGFR mutation-positive non-smallcell lung cancer (OPTIMAL, CTONG-0802): a multicentre, open-label, randomised, phase 3 study. Lancet Oncol. 2011;12:735-742. doi:10.1016/ S1470-2045(11)70184-X

7. Ohashi K, Maruvka YE, Michor F, et al. Epidermal growth factor receptor tyrosine kinase inhibitor-resistant disease. J Clin Oncol. 2013;31:1070-1080. doi:10.1200/JCO.2012.43.3912

8. Oser MG, Niederst MJ, Sequist LV, et al. Transformation from non-small-cell lung cancer to small-cell lung cancer: molecular drivers and cells of origin. Lancet Oncol. 2015;16:e165-e172. doi:10. 1016/S1470-2045(14)71180-5
9. Jiang SY, Zhao J, Wang MZ, et al. Small-cell lung cancer transformation in patients with pulmonary adenocarcinoma: a case report and review of literature. Medicine (Baltimore). 2016;95:e2752. doi:10.10 97/MD.0000000000002752

10. Huang Z, Xu D, Zhang F, et al. Pro-gastrin-releasing peptide and neuron-specific enolase: useful predictors of response to chemotherapy and survival in patients with small cell lung cancer. Clin Transl Oncol. 2016;18:1019-1025. doi:10.1007/s12094-015-1479-4

11. Cavalieri S, Morelli D, Martinetti A, et al. Clinical implications for pro-GRP in small cell lung cancer. A single center experience. Int J Biol Markers. 2018;33:55-61. doi:10.5301/ijbm.5000305

12. Zhang Y, Li XY, Tang Y, et al. Rapid increase of serum neuron specific enolase level and tachyphylaxis of EGFR-tyrosine kinase inhibitor indicate small cell lung cancer transformation from EGFR positive lung adenocarcinoma? Lung Cancer. 2013;81:302-305.

13. Ono A, Naito T, Ito I, et al. Correlations between serial pro-gastrinreleasing peptide and neuron-specific enolase levels, and the radiological response to treatment and survival of patients with small-cell lung cancer. Lung Cancer. 2012;76:439-444. doi:10.1016/j.lungcan.2011.12.012

14. Okamoto H, Watanabe K, Nishiwaki Y, et al. Phase II study of area under the plasma-concentration-versus-time curve-based carboplatin plus standard-dose intravenous etoposide in elderly patients with small-cell lung cancer. J Clin Oncol. 1999;17:3540-3545. doi:10.12 00/JCO.1999.17.11.3540

15. Hanna N, Bunn PA Jr., Langer C, et al. Randomized phase III trial comparing irinotecan/cisplatin with etoposide/cisplatin in patients with previously untreated extensive-stage disease small-cell lung cancer. J Clin Oncol. 2006;24:2038-2043. doi:10.1200/JCO.2005.04.8595

16. Naito Y, Tamiya A, Tamiya M, et al. Efficacy of nanoparticle albumin-bound paclitaxel regimens for relapsed small cell lung cancer: a retrospective analysis. Medicine (Baltimore). 2017;96:e7884. doi:10.1097/MD.0000000000007884

17. Pujol J-L, Lavole A, Quoix E, et al. Randomized phase II-III study of bevacizumab in combination with chemotherapy in previously untreated extensive small-cell lung cancer: results from the IFCT-0802 trialdagger. Ann Oncol. 2015;26:908-914. doi:10.1093/annonc/mdv065

18. Rindi G, Klersy C, Inzani F, et al. Grading the neuroendocrine tumors of the lung: an evidence-based proposal. Endocr Relat Cancer. 2014;21:1-16. doi:10.1530/ERC-13-0246

19. Niederst MJ, Sequist LV, Poirier JT, et al. RB loss in resistant EGFR mutant lung adenocarcinomas that transform to small-cell lung cancer. Nat Commun. 2015;6:6377. doi:10.1038/ncomms 7377

20. Lee JK, Lee J, Kim S, et al. Clonal history and genetic predictors of transformation into small-cell carcinomas from lung adenocarcinomas. J Clin Oncol. 2017;35:3065-3074. doi:10.1200/JCO.2016.71.9096

21. Miyamoto S, Ikushima S, Ono R, et al. Transformation to small-cell lung cancer as a mechanism of acquired resistance to crizotinib and alectinib. Jpn J Clin Oncol. 2016;46:170-173. doi:10.1093/jjco/hyv173

22. Imakita T, Fujita K, Kanai O, et al. Small cell lung cancer transformation during immunotherapy with nivolumab: a case report. Respir Med Case Rep. 2017;21:52-55. doi:10.1016/j.rmcr.2017.0 3.019

\section{Publish your work in this journal}

OncoTargets and Therapy is an international, peer-reviewed, open access journal focusing on the pathological basis of all cancers, potential targets for therapy and treatment protocols employed to improve the management of cancer patients. The journal also focuses on the impact of management programs and new therapeutic agents and protocols on patient perspectives such as quality of life, adherence and satisfaction. The manuscript management system is completely online and includes a very quick and fair peer-review system, which is all easy to use. Visit http://www.dovepress.com/ testimonials.php to read real quotes from published authors. 